\title{
Electronic properties of Mn-decorated silicene on hexagonal boron nitride
}

\author{
T. P. Kaloni, ${ }^{1}$ S. Gangopadhyay, ${ }^{2}$ N. Singh, ${ }^{1}$ B. Jones, ${ }^{2}$ and U. Schwingenschlögl ${ }^{1, *}$ \\ ${ }^{1}$ PSE Division, KAUST, Thuwal 23955-6900, Kingdom of Saudi Arabia \\ ${ }^{2}$ IBM Almaden Research Center, San Jose, California 95120-6099, USA
}

(Received 1 October 2013; published 17 December 2013)

\begin{abstract}
We study silicene on hexagonal boron nitride, using first-principles calculations. Since hexagonal boron nitride is semiconducting, the interaction with silicene is weaker than for metallic substrates. It therefore is possible to open a $50 \mathrm{meV}$ band gap in the silicene. We further address the effect of Mn decoration by determining the onsite Hubbard interaction parameter, which turns out to differ significantly for decoration at the top and hollow sites. The induced magnetism in the system is analyzed in detail.
\end{abstract}

DOI: $10.1103 /$ PhysRevB.88.235418

PACS number(s): 73.20.At, 75.75.Lf

\section{INTRODUCTION}

Silicon based nanostructures, such as two-dimensional silicene (an analog to graphene) and silicene nanoribbons, are currently attracting the interest of many researchers due to materials properties that are similar to but richer than those of graphene. ${ }^{1,2}$ Moreover, they are advantageous to carbon based nanostructures, as they can be expected to be compatible with the existing semiconductor industry. It is observed that the electronic band structure of silicene shows a linear dispersion around the Dirac point, like graphene, and hence is a candidate for applications in nanotechnology. Due to an enhanced spin orbit coupling a band gap $1.55 \mathrm{meV}$ is opened. ${ }^{3}$ A mixture of $s p^{2}$ and $s p^{3}$-type bonding results in a buckled structure, which leads to an electrically tunable band gap. ${ }^{4,5}$ First-principles geometry optimization and phonon calculations as well as temperature dependent molecular dynamics simulations predict a stable low-buckled structure. ${ }^{6}$ Moreover, stability of silicene under biaxial tensile strain has been predicted up to $17 \%$ strain. $^{7}$

Silicene and its derivatives experimentally have been grown on $\mathrm{Ag}$ and $\mathrm{ZrB}_{2}$ substrates, ${ }^{8-10}$ though there is still discussion about the quality of the results. ${ }^{11} \mathrm{On}$ a $\mathrm{ZrB}_{2}$ thin film an asymmetric buckling due to the interaction with the substrate is found, which opens a band gap. In general, transition metal decorated graphene has been studied extensively both in experiment and theory. It has been predicted that $5 d$ transition metal atoms show unique properties with topological transport effects. The large spin orbit coupling of $5 d$ transition metal atoms together with substantial magnetic moments leads to a quantum anomalous Hall effect. ${ }^{12}$ A model study also has predicted the quantum anomalous Hall effect for transition metal decorated silicene nanoribbons. ${ }^{13}$ Energy arguments indicate that transition metal atoms bond to silicene much stronger than to graphene. As a result, a layer by layer growth of transition metals could be possible on silicene. ${ }^{14}$

The deposition of isolated transition metal atoms on layers of hexagonal boron nitride on a $\mathrm{Rh}(111)$ substrate has been studied in Ref. 15. The authors have demonstrated a reversible switching between two states with controlled pinning and unpinning of the hexagonal boron nitride from the metal substrate. In the first state the interaction of the hexagonal boron nitride is reduced, which leads to a highly symmetric ring in scanning tunneling microscopy images, while the second state is imaged as a conventional adatom and corresponds to normal interaction. Motivated by this work, we present in the following a first-principles study of the transition metal decoration of silicene on hexagonal boron nitride. We will first address the interaction with the substrate and then will deal with the electronic and magnetic properties of the Mn decorated system.

\section{COMPUTATIONAL DETAILS}

All calculations have been carried out using density functional theory in the generalized gradient approximation. We employ the QUANTUM-ESPRESSO package, ${ }^{16}$ taking into account the van-der-Waals interaction. ${ }^{17}$ The calculations are performed with a plane wave cutoff energy of $816 \mathrm{eV}$, where a Monkhorst-Pack $8 \times 8 \times 1 \mathrm{k}$-mesh is used to optimize the crystal structure and to obtain the self-consistent electronic structure. The atomic positions are relaxed until an energy convergence of $10^{-7} \mathrm{eV}$ and a force convergence of $0.001 \mathrm{eV} / \AA$ are reached. To study the interaction of the silicene with the substrate, we employ a supercell consisting of a $2 \times 2$ supercell of silicene on top of a $3 \times 3$ supercell of hexagonal boron nitride. We have tested the convergence of the results with respect to the thickness of the substrate by taking into account 2, 3, 4, and 6 atomic layers of $h$-BN, finding only minor differences (in particular concerning the splitting and position of the Dirac cone) because of the inert nature of the substrate. A thin substrate consequently turns out to be fully sufficient in the calculations. Moreover, the $2 \times 2$ supercell of silicene fits well on the $3 \times 3$ supercell of the substrate with a lattice mismatch of only $2.8 \%$. When we consider Mn decorated silicene we use a larger supercell that contains $16 \mathrm{Si}$ in a layer over $18 \mathrm{~B}$ and $18 \mathrm{~N}$. While the onsite Hubbard parameter for $3 d$ transition metal atoms is known to be several $\mathrm{eV}$, we explicitly calculate the value in the present study for the different adsorption sites in order to obtain accurate results for the electronic and magnetic properties of the Mn decorated system.

In general, the lattice mismatch of $2.8 \%$ between silicene and hexagonal boron nitride can be expected to be small enough to avoid experimental problems with a controlled growth. Moreover, accurate measurements of materials properties can be difficult to achieve on metallic substrates, whereas the interaction is reduced on semiconducting substrates. Our calculated binding energy for the interface between silicene and hexagonal boron nitride is only $100 \mathrm{meV}$ per $\mathrm{Si}$ atom, 
(a)

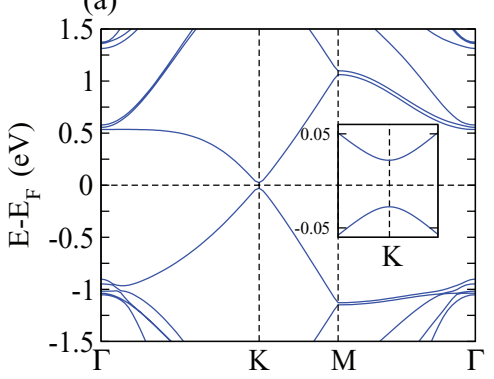

(b)

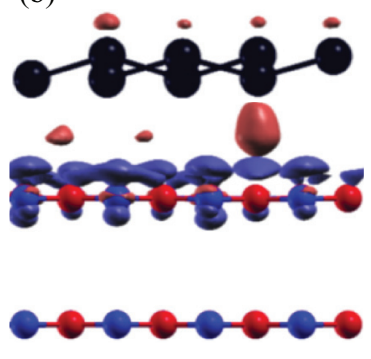

FIG. 1. (Color online) (a) Electronic band structure and (b) charge transfer for silicene on a bilayer of $\mathrm{BN}$ (side view). The isosurfaces correspond to isovalues of $\pm 5 \times 10^{-4}$ electrons $/ \AA^{3}$. The black, blue, and red spheres denote $\mathrm{Si}, \mathrm{N}$, and $\mathrm{B}$ atoms, respectively. Red and blue isosurfaces refer to positive and negative charge transfer. Note the significant charge transfer of the Si closest to the BN.

as compared to typically $500 \mathrm{meV}$ per $\mathrm{Si}$ atom for an interface to a metallic substrate. Experimental realizations of graphene based electronic devices using hexagonal boron nitride as substrate on a $\mathrm{Si}$ wafer support are subject to various limitations, such as a poor on/off ratio. ${ }^{18}$ However, on this substrate graphene exhibits the highest mobility ${ }^{19}$ and a sizable band gap. ${ }^{20-22}$ Since silicene resembles the structure of graphene, synthesis on hexagonal boron nitride thus has great potential.

The structural arrangement of the system under study is depicted in Fig. 1(b), together with the charge redistribution introduced by the interaction with the substrate. We obtain $\mathrm{Si}-\mathrm{Si}$ bond lengths of 2.24 to $2.26 \AA$ and a buckling of 0.48 to $0.54 \AA$, which is slightly higher than in free-standing silicene. $^{3,6}$ For the angle between the Si-Si bonds and the normal of the silicene sheet we observe values of $113^{\circ}$ to $115^{\circ}$, again close to the findings for free-standing silicene $\left(116^{\circ}\right)$. The optimized distance between the silicene and hexagonal boron nitride sheets forming the interface turns out to be $3.57 \AA$, which is similar to the distance at the contact between graphene and hexagonal boron nitride. In addition, the interlayer distance within the hexagonal boron nitride amounts to be $3.40 \AA$, whereas in a bilayer configuration values of 3.30 to $3.33 \AA$ have been reported. ${ }^{23,24}$

The interaction between silicene and hexagonal boron nitride recently has been addressed by Liu and coworkers, ${ }^{25}$ who have reported a perturbation of the Dirac cone with an energy gap of $4 \mathrm{meV}$. This study has taken into account only a single layer of hexagonal boron nitride as substrate, so that a more realistic description may yield a different result. Indeed, we observe a perturbed Dirac cone with an energy gap of $50 \mathrm{meV}$ in the band structure shown in Fig. 1(a). The $\pi$ and $\pi^{*}$ bands forming the Dirac cone are due to the $p_{z}$ orbitals of the $\mathrm{Si}$ atoms, while the bands related to the $\mathrm{B}$ and $\mathrm{N}$ atoms are located about $0.5 \mathrm{eV}$ above and $1 \mathrm{eV}$ below the Fermi energy. We find a small but finite charge redistribution across the interface to the substrate; see the charge density difference isosurfaces plotted in Fig. 1(b). As a result the Dirac cone is perturbed and the $50 \mathrm{meV}$ energy gap is realized, which can be interesting for nanoelectronic device applications, in particular because an external electric field can be used to tune the gap. The isosurface plot also demonstrates that the Si atom closest

to a B atom is subject to the strongest charge transfer, while for all other $\mathrm{Si}$ atoms charge transfer effects are subordinate due to longer interatomic distances.

\section{MN DECORATION}

The possible decoration sites for a $\mathrm{Mn}$ atom on silicene can be classified as top, bridge, and hollow. Decoration at the bridge site is not considered in the following because the $\mathrm{Mn}$ atom immediately transfers to the top site. A side view of the relaxed structure for $\mathrm{Mn}$ decoration at the top site is given in Fig. 2(a), together with a spin density map. We obtain the onsite interaction parameter using a constraint method, ${ }^{26}$ and calculate the values of $3.8 \mathrm{eV}$ for the $\mathrm{Mn}$ atom at the top site and $4.5 \mathrm{eV}$ for the $\mathrm{Mn}$ atom at the hollow site. For the top site configuration, structural optimization reveals that the $\mathrm{Mn}$ atom moves close to an original Si position and thereby strongly displaces this $\mathrm{Si}$ atom, resulting in a short $\mathrm{Mn}-\mathrm{Si}$ bond length of $2.43 \AA$. Moreover, the $\mathrm{Mn}$ atom is bound to three $\mathrm{Si}$ atoms with equal bond lengths of $2.45 \AA$. Si-Si bond lengths of 2.24 to $2.28 \AA$ are observed, which corresponds to a slight modification as compared to the pristine configuration. The buckling of the silicene, on the other hand, is strongly altered, now amounting to 0.45 to $0.67 \AA$. Accordingly, angles of $113^{\circ}$ to $117^{\circ}$ are found between the $\mathrm{Si}-\mathrm{Si}$ bonds and the normal of the silicene sheet. The height of the Mn atom above the silicene sheet is $1.30 \AA$. Finally, we note that the separation between the atomic layers of the hexagonal boron nitride is virtually not modified by the Mn decoration.

A side view of the relaxed structure for Mn decoration on the hollow site is shown in Fig. 2(b). In this case, the Mn

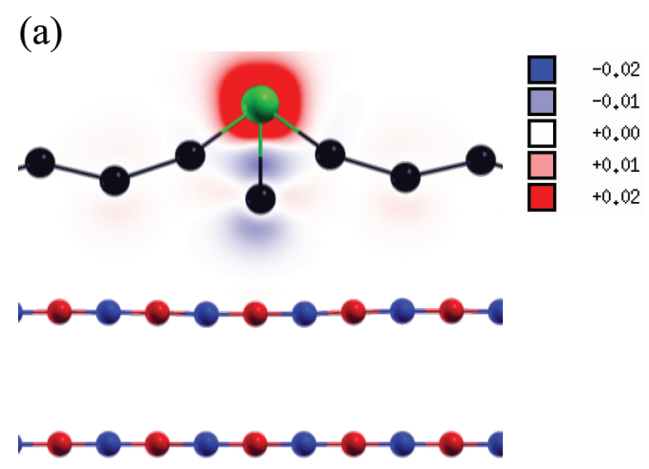

(b)
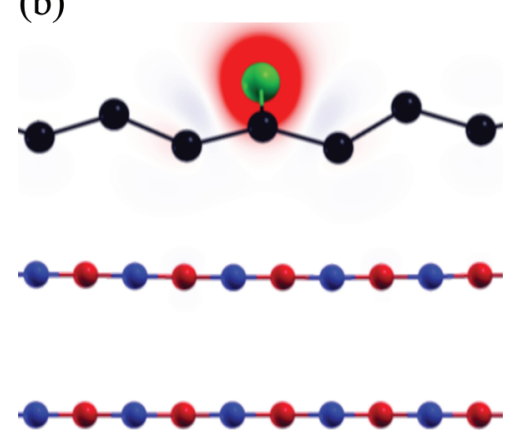

FIG. 2. (Color online) The spin density map for silicene decorated by $\mathrm{Mn}$ at the (a) top and (b) hollow site of the $h$-BN substrate. The hollow site is energetically favorable. 

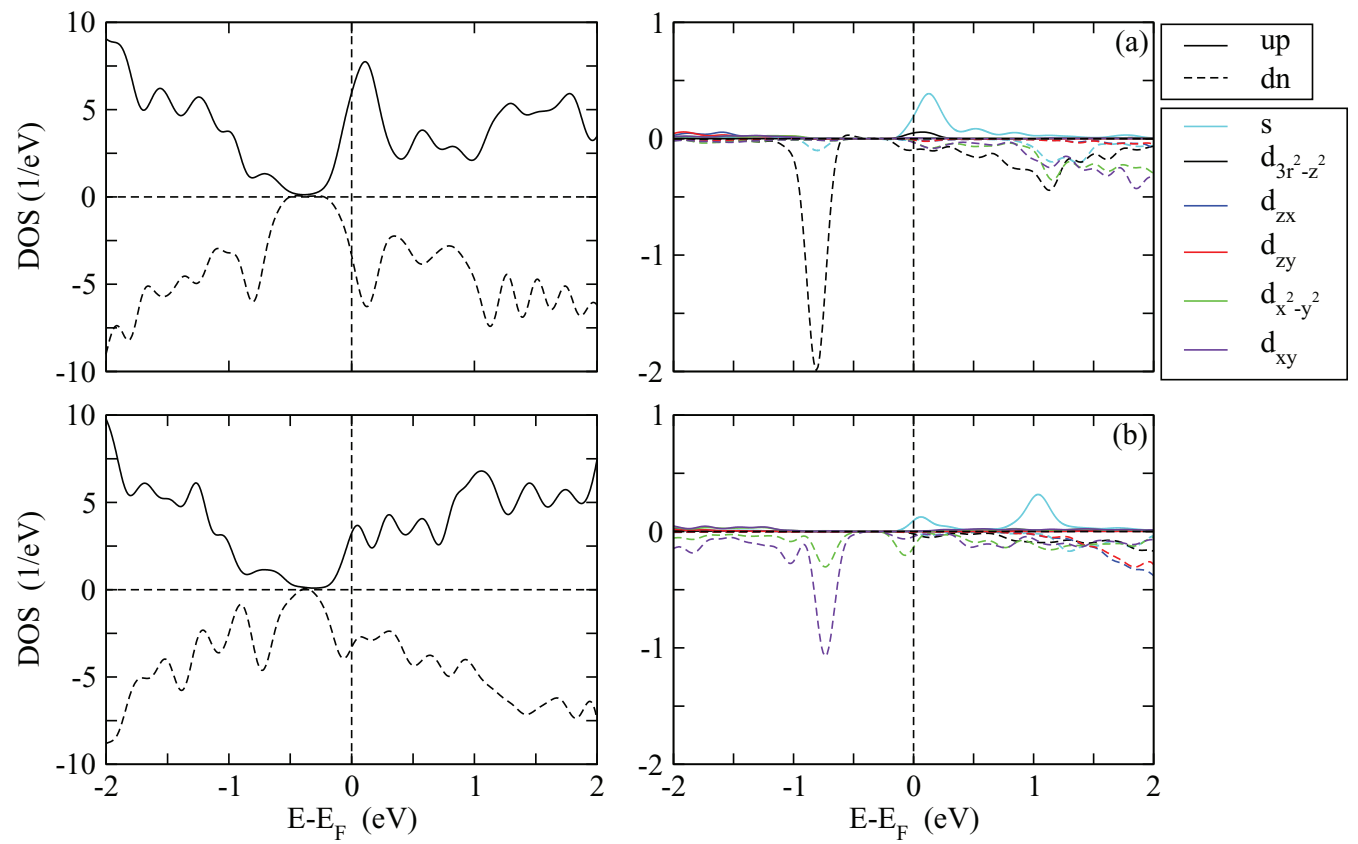

FIG. 3. (Color online) Total (left) and Mn partial (right) densities of states of silicene decorated by Mn at the (a) top and (b) hollow site of the $h$-BN substrate.

atom does not displace a specific Si atom but stays close to the center of the Si hexagon. It is bound equally to the neighboring $\mathrm{Si}$ atoms with bond lengths of $2.40 \AA$ to the upper three and $2.77 \AA$ to the lower three $\mathrm{Si}$ atoms. A buckling of 0.46 to $0.62 \AA, \mathrm{Si}$-Si bond lengths of 2.23 to $2.28 \AA$, and angles to the normal of $112^{\circ}-117^{\circ}$ are obtained. The $\mathrm{Mn}$ atom is located $1.01 \AA$ above the silicene sheet and the separation between the atomic layers in the hexagonal boron nitride is slightly increased to $3.44 \AA$. In contrast, the distance between silicene and substrate here amounts to $3.55 \AA$ and thus is significantly larger than in the case of decoration at the top site, because in the latter case one Si atom is displaced from the silicene sheet, which modifies the distance to the substrate. The calculated total energies indicate that decoration at the hollow site is by $33 \mathrm{meV}$ favorable as compared to decoration at the top site.

\section{ELECTRONIC STRUCTURE}

We find total magnetic moments of 4.56 and $3.50 \mu_{B}$ per supercell for Mn decoration at the top and hollow sites, a reduction of spin from the free $\mathrm{Mn}$ value of 5.0 unpaired electrons. The magnetization reduction is notable on the hollow site, which can be seen from Figs. 2 and 3 to involve greater immersion in and hybridization with the Si than the top site. By far the largest contribution to the magnetic moment comes from the Mn atom and only small moments are induced on the Si atoms. This can be clearly seen in the spin density maps presented in Figs. 2(a) and 2(b). For Mn decoration at the top site we obtain a Mn moment of $4.40 \mu_{B}$ and a total of $0.16 \mu_{B}$ from all the $\mathrm{Si}$ atoms, whereas for decoration at the hollow site the Mn moment amounts to $4.16 \mu_{B}$ and the $\mathrm{Si}$ atoms contribute a total $-0.66 \mu_{B}$. These results indicate that the $\mathrm{Mn}$ and $\mathrm{Si}$ moments are ordered ferromagnetically and antiferromagnetically for decoration at the top and hollow sites, respectively.
In Fig. 3 we address the density of states (DOS) for decoration at the (a) top and (b) hollow sites. The left panel of the figure shows the total DOS and the right panel the partial DOSs of the Mn $3 d$ and $4 s$ orbitals. In contrast to pristine silicene (band gap of $1.55 \mathrm{meV}^{3}$ ), the DOSs show a region without states around $0.5 \mathrm{eV}$ below the Fermi energy. This observation corresponds to an $n$ doping due to the mentioned charge transfer from Mn to silicene. Closer inspection of the partial DOSs for decoration at the top site shows that the spin majority $s$ and $d_{3 r^{2}-z^{2}}$ as well as the spin minority $d_{3 r^{2}-z^{2}}$, $d_{x^{2}-y^{2}}$, and $d_{x y}$ states contribute in the vicinity of the Fermi energy, while there are essentially no contributions from the $d_{z x}$ and $d_{z y}$ states. A sharp Mn peak is observed about $0.8 \mathrm{eV}$, which is due to the spin minority $d_{3 r^{2}-z^{2}}$ states. For decoration at the hollow site almost exclusively the spin majority $s$ and spin minority $d_{x^{2}-y^{2}}$ and $d_{x y}$ states contribute around the Fermi energy. Two less pronounced DOS peaks appear $0.75 \mathrm{eV}$ below the Fermi energy, contributed by the spin minority $d_{x y}$ and $d_{x^{2}-y^{2}}$ states.

\section{CONCLUSION}

In conclusion, we have employed density functional theory to discuss the structure and chemical bonding of silicene on hexagonal boron nitride. The interaction results in a band gap of $50 \mathrm{meV}$. Furthermore, we have calculated the onsite Hubbard interaction parameter for Mn decoration at the top and hollow sites of the silicene, finding values of 3.8 and $4.5 \mathrm{eV}$, respectively. The electronic and magnetic properties of Mn decorated silicene have been studied in detail. In particular, magnetic moments of 3.50 and $4.56 \mu_{B}$, respectively, have been obtained for $\mathrm{Mn}$ decoration at the top and hollow sites. Interestingly, the orientation between the $\mathrm{Mn}$ and induced $\mathrm{Si}$ moments is ferromagnetic in the former and antiferromagnetic in the latter case. 
*udo.schwingenschloegl@physik.uni-augsburg.de

${ }^{1}$ G. G. Guzmán-Verri and L. C. Lew Yan Voon, Phys. Rev. B 76, 075131 (2007).

${ }^{2}$ S. Lebegue and O. Eriksson, Phys. Rev. B 79, 115409 (2009).

${ }^{3}$ C.-C. Liu, W. Feng, and Y. Yao, Phys. Rev. Lett. 107, 076802 (2011).

${ }^{4}$ N. D. Drummond, V. Zólyomi, and V. I. Fal'ko, Phys. Rev. B 85, 075423 (2012).

${ }^{5}$ Z. Ni, Q. Liu, K. Tang, J. Zheng, J. Zhou, R. Qin, Z. Gao, D. Yu, and J. Lu, Nano Lett. 12, 113 (2012).

${ }^{6}$ S. Cahangirov, M. Topsakal, E. Aktürk, H. Sahin, and S. Ciraci, Phys. Rev. Lett. 102, 236804 (2009).

${ }^{7}$ T. P. Kaloni, Y. C. Cheng, and U. Schwingenschlögl, J. Appl. Phys. 113, 104305 (2013).

${ }^{8}$ P. De Padova, C. Quaresima, C. Ottaviani, P. M. Sheverdyaeva, P. Moras, C. Carbone, D. Topwal, B. Olivieri, A. Kara, H. Oughaddou, B. Aufray, and G. Le Lay, Appl. Phys. Lett. 96, 261905 (2010).

${ }^{9}$ P. Vogt, P. De Padova, C. Quaresima, J. Avila, E. Frantzeskakis, M. C. Asensio, A. Resta, B. Ealet, and G. Le Lay, Phys. Rev. Lett. 108, 155501 (2012).

${ }^{10}$ A. Fleurence, R. Friedlein, T. Ozaki, H. Kawai, Y. Wang, and Y. Yamada-Takamura, Phys. Rev. Lett. 108, 245501 (2012).

${ }^{11}$ C.-L. Lin, R. Arafune, K. Kawahara, M. Kanno, N. Tsukahara, E. Minamitani, Y. Kim, M. Kawai, and N. Takagi, Phys. Rev. Lett. 110, 076801 (2013).

${ }^{12}$ H. Zhang, C. Lazo, S. Blügel, S. Heinze, and Y. Mokrousov, Phys. Rev. Lett. 108, 056802 (2012).
${ }^{13}$ M. Ezawa, Phys. Rev. Lett. 109, 055502 (2012).

${ }^{14}$ X. Lin and J. Ni, Phys. Rev. B 86, 075440 (2012).

${ }^{15}$ F. D. Natterer, F. Patthey, and H. Brune, Phys. Rev. Lett. 109, 066101 (2012).

${ }^{16}$ P. Giannozzi et al., J. Phys.: Condens. Matter 21, 395502 (2009).

${ }^{17}$ S. Grimme, J. Comput. Chem. 27, 1787 (2006).

${ }^{18}$ K. Kim, J.-Y. Choi, T. Kim, S.-H. Cho, and H.-J. Chung, Nature (London) 479, 338 (2011).

${ }^{19}$ C. R. Dean, A. F. Young, I. Meric, C. Lee, L. Wang, S. Sorgenfrei, K. Watanabe, T. Taniguchi, P. Kim, K. L. Shepard, and J. Hone, Nat. Nanotechnol. 5, 722 (2010).

${ }^{20}$ S. Y. Zhou, G.-H. Gweon, A. V. Fedorov, P. N. First, W. A. de Heer, D.-H. Lee, F. Guinea, A. H. Castro Neto, and A. Lanzara, Nat. Mater. 6, 770 (2007).

${ }^{21}$ R. Quhe, J. Zheng, G. Luo, Q. Liu, R. Qin, J. Zhou, D. Yu, S. Nagase, W.-N. Mei, Z. Gao, and J. Lu, NPG Asia Materials 4, 1 (2012).

${ }^{22}$ T. P. Kaloni, Y. C. Cheng, and U. Schwingenschlögl, J. Mater. Chem. 22, 919 (2012).

${ }^{23}$ A. Marini, P. Garcia-Gonzalez, and A. Rubio, Phys. Rev. Lett. 96, 136404 (2006).

${ }^{24}$ Y. Shi, C. Hamsen, X. Jia, K. K. Kim, A. Reina, M. Hofmann, A. L. Hsu, K. Zhang, H. Li, Z.-Y. Juang, M. S. Dresselhaus, L.-J. Li, and J. Kong, Nano Lett. 10, 4134 (2010).

${ }^{25}$ H. Liu, J. Gao, and J. Zhao, J. Phys. Chem. C 117, 10353 (2013).

${ }^{26}$ G. K. H. Madsen and P. Novák, Europhys. Lett. 69, 777 (2005). 